\title{
Improved Estimation of Clock Offset in Sensor Networks
}

\author{
Qasim M. Chaudhari*, Erchin Serpedin* and Yik-Chung $\mathrm{Wu}^{\dagger}$ \\ *Department of Electrical and Computer Engineering, \\ Texas A\&M University, \\ College Station, TX 77843-3128, USA. \\ Email:\{qasim,serpedin\}@ece.tamu.edu. \\ ${ }^{\dagger}$ Department of Electrical and Electronic Engineering \\ The University of Hong Kong, \\ Pokfulam Road, Hong Kong. \\ Email: ycwu@eee.hku.hk.
}

\begin{abstract}
Clock synchronization is an important issue for the design of a network composed of small sensor nodes. Based on the two-way timing message exchange mechanism and assuming an exponential network delay distribution, many analytical results have been presented in the literature by applying the techniques from statistical signal processing. This paper derives the minimum variance unbiased estimator for the clock offset for both symmetric and asymmetric exponential delay cases. For the asymmetric delays, it is shown to be a function of both the minimum and the mean link delays. This result is a very significant contribution since only the minimum link delay observations have been used to estimate the clock offset in the past. For the symmetric case, it is shown to coincide with the maximum likelihood estimator. In addition, the result is also applicable to clock synchronization problem in general computer networks.
\end{abstract}

\section{INTRODUCTION}

Clock synchronization is an important problem in any distributed networks, and this fact is particularly true for wireless sensor networks. In such networks, the power consumption is strictly constrained and hence it becomes vital to extract the maximum amount of information from the same set of communicated messages. Today various applications have emerged for which WSNs can be deployed for efficient results, e.g., fire detection, habitat monitoring, security, object tracking, etc. Most of these applications such as object tracking, data fusion, security protocols, etc. require the nodes of the sensor network to be synchronized in time. In addition, since energy is the scarcest resource in WSNs, a nice technique to conserve energy is to deploy coordinated turning on and off of radios in sensor nodes. If the nodes are time synchronized with each other, the efficient duty cycling operation of coordinated sleep and wakeup modes can be enabled which hugely boosts the lifetime of the network due to the nominal power consumption during their sleep mode.

Many clock synchronization protocols have been proposed in the past by the researchers. The Network Time Protocol (NTP) [1] is a protocol for synchronizing the clocks of computer systems over packet-switched, variable-latency data networks and it represents the Internet standard for time synchronization. For ad-hoc communication networks, the time synchronization protocol [2] represented one of the significant contributions in this area. In the realm of WSNs, the clock synchronization protocols of particular note are Reference Broadcast Synchronization (RBS [3]), Timing Synch Protocol for Sensor Networks (TPSN [4]) and Time Diffusion Protocol (TDP [5]).

Based on the two-way timing message exchange mechanism and assuming an exponential network delay distribution, [6] presented some analytical results by applying the techniques from statistical signal processing. Assuming known fixed delays and known symmetric exponential mean link delays, [6] concluded that the MLE of the clock offset does not exist in this scenario. However, in [7], for an unknown fixed delay, irrespective of the symmetric exponential mean link delay being known or unknown, the MLE of the clock offset was successfully derived. In this paper, the Minimum Variance Unbiased Estimate (MVUE) for the clock offset is derived for both symmetric and asymmetric network link delays. It is shown to be a function of both mean and minimum link delays for the asymmetric delays, while it coincides with the MLE in the symmetric case. This finding is very important since only the minimum link delays have been used in the previous works to estimate the clock offset. Also, the result has its application in traditional computer networks too, where it can be used to improve the quality of the NTP clock offset estimate, where the difference between the minimum out of eight different forward and reverse link delays is employed.

\section{SySTEM MODEL}

For a sender-receiver protocol, the timing message exchange mechanism between two nodes presenting both clock offset and skew is depicted in Fig. 1. Node $c$ sends a synchronization message to node $d$ with its current timestamp $s_{k}^{c}$ which is recorded by node $d$ at its current time $r_{k}^{d}$ at the reception of this message. The second round of this message exchange mechanism is completed by sending and reception of $s_{k}^{d}$ and $r_{k}^{c}$, respectively (see Fig. 1). This process between the two nodes is repeated $N$ times, where $N$ stands for the required number of samples. It should be noted that $N$ is a function of the target synchronization accuracy and the price the protocol is willing to invest in the form of network resources.

Based on the above pairwise synchronization message exchange mechanism, the clock offset measurement model can 


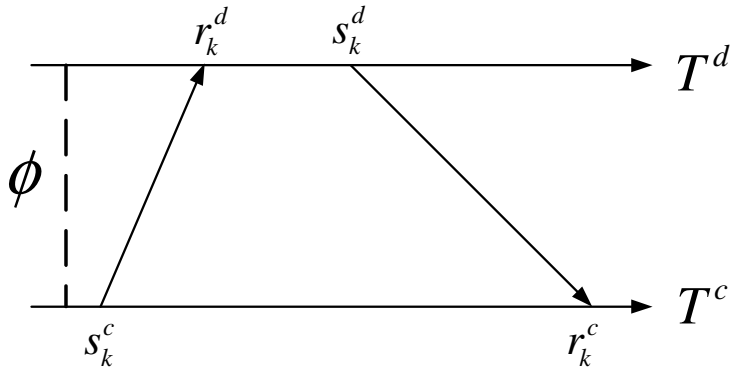

Fig. 1. A sender-receiver timing message exchange paradigm.

be represented in terms of these two equations:

$$
\begin{aligned}
& r_{k}^{d}=s_{k}^{c}+\delta+\phi+X_{k}, \\
& r_{k}^{c}=s_{k}^{d}+\delta-\phi+Y_{k} .
\end{aligned}
$$

Simplifying the notation, the above equations can be rewritten as

$$
\begin{aligned}
U_{k} & =\delta+\phi+X_{k}, \\
V_{k} & =\delta-\phi+Y_{k},
\end{aligned}
$$

where $U_{k} \triangleq r_{k}^{d}-s_{k}^{c}$ and $V_{k} \triangleq r_{k}^{c}-s_{k}^{d}$. The quantity $\delta$ symbolizes the fixed portions of the delays which can be asymmetric or symmetric for each direction, $X_{i}$ and $Y_{i}$ denote the variable portions of delays and assume exponential distributions with means $\alpha$ and $\beta$, respectively, and $\phi$ stands for the clock offset of node $d$ with respect to node $c$. Network delay modeling has always been an active research topic for the last decades. There is extensive discussion in the literature about why the network delays are usually modeled by the exponential distribution such as [6] and the references cited therein.

In [7], it was argued that for an unknown $\delta$, irrespective of the symmetric exponential distribution mean $\alpha=\beta \triangleq \lambda$ being known or unknown, the MLE of the vector parameter $\mathbf{\Phi}_{\mathbf{M L E}}^{\mathbf{S}}=[\delta \phi \lambda]$ is given by

$$
\hat{\mathbf{\Phi}}_{\mathrm{MLE}}^{\mathbf{S}}=\frac{1}{2}\left[\begin{array}{c}
U_{(1)}+V_{(1)} \\
U_{(1)}-V_{(1)} \\
\bar{U}+\bar{V}-\left(U_{(1)}+V_{(1)}\right)
\end{array}\right],
$$

where $U_{(1)}$ and $V_{(1)}$ denote the minimum order statistics, and $\bar{U}$ and $\bar{V}$ represent the sample average of the data $\left\{U_{k}\right\}_{k=1}^{N}$ and $\left\{V_{k}\right\}_{k=1}^{N}$, respectively. When $\lambda$ is known, the MLE of $\{\delta, \phi\}$ remains the same.

\section{Estimation of Clock OfFSET}

In parameter estimation, very often the ultimate goal is to find the estimator that achieves the minimum MSE, and it is usually the criterion of choice. However, it is well known in theory that the optimal MSE estimators are usually not realizable. Since the MSE is the sum of estimator variance and squared bias, a technique chosen to attain realizable yet best estimators is to constrain the bias to be zero (since the dependance of minimum MSE estimator on the unknown parameter typically comes from the bias). Therefore, restricting the possible estimators to be unbiased and then finding the estimator with the smallest variance for all values of the unknown parameter yields the optimal solution within the class of unbiased estimators. Therefore, we will resort on the concept of MVUE.

In this paper, the MVUE is derived based on the RaoBlackwell-Lehmann-Scheffé theorem. First, the likelihood function should be factored according to Neymann-Fisher factorization theorem yielding the sufficient statistics $\mathbf{T}$. Then, it should be determined if the sufficient statistics are complete. Finally, either for any unbiased estimator $\check{\theta}, \hat{\theta}=E[\check{\theta} \mid \mathbf{T}]$ should be evaluated, or a function $g(\mathbf{T})$ of the sufficient statistics should be found such that $\hat{\theta}=g(\mathbf{T})$ is an unbiased estimator, producing $\hat{\theta}$ as the MVUE. The approach that we will follow next relies on similar steps.

\section{A. Asymmetric Link Delays}

Starting with the asymmetric case, the likelihood function for the clock offset as a function of observations $\left\{U_{k}\right\}_{k=1}^{N}$ and $\left\{V_{k}\right\}_{k=1}^{N}$ is given by

$$
\begin{aligned}
L(\delta, \phi, \alpha, \beta)= & \alpha^{-N} e^{-\frac{1}{\alpha} \sum_{k=1}^{N}\left\{U_{k}-\delta-\phi\right\}} u\left[U_{(1)}-\delta-\phi\right] . \\
& \beta^{-N} e^{-\frac{1}{\beta} \sum_{k=1}^{N}\left\{V_{k}-\delta+\phi\right\}} u\left[V_{(1)}-\delta+\phi\right],
\end{aligned}
$$

where $u[\cdot]$ denotes the unit step function. Now (2) can be factored as

$$
\begin{array}{r}
L(\delta, \phi, \alpha, \beta)=g_{1}\left(\sum_{k=1}^{N} U_{k}, \delta, \phi, \alpha\right) g_{2}\left(\sum_{k=1}^{N} V_{k}, \delta, \phi, \beta\right) . \\
g_{3}\left(U_{(1)}, \delta, \phi\right) g_{4}\left(V_{(1)}, \delta, \phi\right) h_{1}\left(U_{k}, V_{k}\right)
\end{array}
$$

where

$$
\begin{array}{r}
g_{1}\left(\sum_{k=1}^{N} U_{k}, \delta, \phi, \alpha\right)=\alpha^{-N} e^{-\frac{1}{\alpha} \sum_{k=1}^{N}\left(U_{k}-\delta-\phi\right)}, \\
g_{2}\left(\sum_{k=1}^{N} V_{k}, \delta, \phi, \beta\right)=\beta^{-N} e^{-\frac{1}{\beta} \sum_{k=1}^{N}\left(V_{k}-\delta+\phi\right)}, \\
g_{3}\left(U_{(1)}, \delta, \phi\right)=u\left[U_{(1)}-\delta-\phi\right], . \\
g_{4}\left(V_{(1)}, \delta, \phi\right)=u\left[V_{(1)}-\delta+\phi\right], h_{1}\left(U_{k}, V_{k}\right)=1 .
\end{array}
$$

In the above relations, $h_{1}\left(U_{k}, V_{k}\right)$ is independent of the unknown vector parameter $\mathbf{\Phi}_{\mathbf{A}}=\left[\begin{array}{llll}\delta & \phi & \alpha & \beta\end{array}\right]^{T}$, whereas $g_{1}\left(\sum_{k=1}^{N} U_{k}, \delta, \phi, \alpha\right), g_{2}\left(\sum_{k=1}^{N} V_{k}, \delta, \phi, \beta\right), g_{3}\left(U_{(1)}, \delta, \phi\right)$ and $g_{4}\left(V_{(1)}, \delta, \phi\right)$ are functions depending on the data through $\mathbf{T}=\left\{\sum_{k=1}^{N} U_{k}, U_{(1)}, \sum_{k=1}^{N} V_{k}, V_{(1)}\right\}$. Therefore, according to Neyman-Fisher factorization theorem, $\mathbf{T}$ is a sufficient statistic for $\boldsymbol{\Phi}_{\mathbf{A}}$.

Since $\operatorname{dim}(\mathbf{T})=\operatorname{dim}\left(\boldsymbol{\Phi}_{\mathbf{A}}\right)$, it is easier to determine the MVUE directly from $\mathbf{T}$ without having to evaluate $E\left[\check{\mathbf{\Phi}}_{\mathbf{A}} \mid \mathbf{T}\right]$ by finding a $4 \times 1$ vector function $\hat{\boldsymbol{\Phi}}_{\mathbf{A}}$ such that $E\left[\hat{\boldsymbol{\Phi}}_{\mathbf{A}}\right]=\mathbf{\Phi}_{\mathbf{A}}$, provided that $\mathbf{T}$ is a complete sufficient statistic. Finding the probability density function (pdf) of $\mathbf{T}$ is required to prove that $\mathbf{T}$ is complete but the problem of finding this pdf is a little 
complex, because $\sum_{k=1}^{N} U_{k}$ and $U_{(1)}$, and similarly $\sum_{k=1}^{N} V_{k}$ and $V_{(1)}$, are not independent.

The joint pdf of $U_{(1)}, U_{(2)}, \cdots, U_{(N)}$ is given by

$$
\begin{array}{r}
p\left(U_{(1)}, U_{(2)}, \cdots, U_{(N)}\right)=N ! \alpha^{-N} e^{-\frac{1}{\alpha} \sum_{k=1}^{N}\left\{U_{k}-\delta-\phi\right\}} . \\
\prod_{k=1}^{N} u\left[U_{k}-\delta-\phi\right]
\end{array}
$$

whereas the pdf of the minimum order statistic $U_{(1)}$ is also exponential with mean $\alpha / N$. Now consider the transformation

$$
z_{k}=(N-k+1)\left(U_{k}-U_{(k-1)}\right), \quad k=1,2, \cdots, N,
$$

where $U_{(0)}=\delta+\phi$. Since $\sum_{k=1}^{N}\left(U_{k}-\delta-\phi\right)=\sum_{k=1}^{N} z_{k}$ and the Jacobian of the transformation is $N$ !, a substitution in (3) reveals that

$$
p\left(z_{1}, z_{2}, \cdots, z_{N}\right)=\alpha^{-N} e^{-\frac{1}{\alpha} \sum_{k=1}^{N} z_{k}} \cdot \prod_{k=1}^{N} u\left[z_{k}\right]
$$

i.e., $z_{k}$ are independent exponential random variables with similar mean $\alpha$. In addition, since each $z_{k} \sim \exp (\alpha)$, each $z_{k}$ assumes a Gamma distribution $z_{k} \sim \Gamma(1, \alpha)$, too. Using the relationship $\sum_{k=1}^{N}\left(U_{k}-U_{(1)}\right)=\sum_{k=2}^{N} z_{k}$, and the fact that each of $z_{2}, z_{3}, \cdots, z_{N}$ is independent of $z_{1}$ (and hence of $U_{(1)}$, since $\left.z_{1}=N\left(U_{(1)}-\delta-\phi\right)\right), \sum_{k=1}^{N}\left(U_{k}-U_{(1)}\right) \sim \Gamma(N-1, \alpha)$ and is independent of $U_{(1)}$.

By a similar reasoning, it can be deduced that $\sum_{k=1}^{N}\left(V_{k}-\right.$ $\left.V_{(1)}\right) \sim \Gamma(N-1, \beta)$ and is independent of $V_{(1)}$. Therefore, the one-to-one function $\mathbf{T}^{\prime}=\left\{\sum_{k=1}^{N}\left(U_{k}-\right.\right.$ $\left.\left.U_{(1)}\right), U_{(1)}, \sum_{k=1}^{N}\left(V_{k}-V_{(1)}\right), V_{(1)}\right\}$ of $\mathbf{T}$ is also sufficient for estimating $\boldsymbol{\Phi}_{\mathbf{A}}$ because the sufficient statistics are unique within one-to-one transformations [8]. Consequently, $\mathbf{T}^{\prime}$ comprises of four independent random variables, that in terms of the three-parameter Gamma distribution assume the distributions:

$$
\begin{array}{r}
r=\sum_{k=1}^{N}\left(U_{k}-U_{(1)}\right) \sim \Gamma(N-1, \alpha, 0), \\
s=\sum_{k=1}^{N}\left(V_{k}-V_{(1)}\right) \sim \Gamma(N-1, \beta, 0), \\
U_{(1)} \sim \Gamma(1, \alpha / N, \delta+\phi), V_{(1)} \sim \Gamma(1, \beta / N, \delta-\phi) .
\end{array}
$$

Note that the domains of $r$ and $s$ are controlled by $U_{(1)}$ and $V_{(1)}$, respectively. Next, it has to be checked whether $\mathbf{T}^{\prime}$, or equivalently $\mathbf{T}$, is complete. Completeness implies that there is but one function of $\mathbf{T}$ that is unbiased. Let $g\left(\mathbf{T}^{\prime}\right)$ be a function of $\mathbf{T}^{\prime}$ such that $E\left[g\left(\mathbf{T}^{\prime}\right)\right]=\mathbf{\Phi}_{\mathbf{A}}$. Suppose that there exists another function $h$ for which $E\left[h\left(\mathbf{T}^{\prime}\right)\right]=\boldsymbol{\Phi}_{\mathbf{A}}$ is also true. Then,

$$
E\left[g\left(\mathbf{T}^{\prime}\right)-h\left(\mathbf{T}^{\prime}\right)\right]=E\left[\pi\left(\mathbf{T}^{\prime}\right)\right]=0 \quad \forall \mathbf{\Phi}_{\mathbf{A}}
$$

where $\pi\left(\mathbf{T}^{\prime}\right) \triangleq g\left(\mathbf{T}^{\prime}\right)-h\left(\mathbf{T}^{\prime}\right)$ and the expectation is taken with respect to $p_{\mathbf{T}^{\prime}}\left(\mathbf{T}^{\prime} ; \boldsymbol{\Phi}_{\mathbf{A}}\right)$. As a result,

$$
\begin{gathered}
\iiint \int_{R_{U_{(1)}}, V_{(1)}} \pi\left(r, U_{(1)}, s, V_{(1)}\right) \cdot \frac{(\alpha \beta)^{-(N-1)}}{\{\Gamma(N-1)\}^{2}}(r s)^{N-2} e^{-\frac{r}{\alpha}-\frac{s}{\beta}} . \\
\frac{N^{2}}{\alpha \beta} e^{-\frac{N}{\alpha}\left\{U_{(1)}-\delta-\phi\right\}-\frac{N}{\beta}\left\{V_{(1)}-\delta+\phi\right\}} d r d U_{(1)} d s d V_{(1)}=0,
\end{gathered}
$$

which is true for all $\mathbf{\Phi}_{\mathbf{A}}$ and where $R_{U_{(1)}, V_{(1)}}$ is the region defined by $u\left[U_{(1)}-\delta-\phi\right]$ and $u\left[V_{(1)}-\delta-\phi\right]$. The above relation can be expressed as

$$
\begin{array}{r}
\int_{-\infty}^{\infty} \int_{-\infty}^{\infty} \int_{-\infty}^{\infty} \int_{-\infty}^{\infty} \pi\left(r, U_{(1)}, s, V_{(1)}\right) \cdot(r s)^{N-2} . \\
e^{-\left\{\frac{r}{\alpha}+\frac{N U_{(1)}}{\alpha}+\frac{s}{\beta}+\frac{N V_{(1)}}{\beta}\right\}} d r d U_{(1)} d s d V_{(1)}=0 \forall \mathbf{\Phi}_{\mathbf{A}}
\end{array}
$$

The expression on the left above is the four-dimensional Laplace transform of the function $\pi\left(\mathbf{T}^{\prime}\right)$. It follows from the uniqueness theorem for two-sided Laplace transform that $\pi\left(\mathbf{T}^{\prime}\right)=0$ almost everywhere, resulting in $g\left(\mathbf{T}^{\prime}\right)=h\left(\mathbf{T}^{\prime}\right)$ and hence there is only one unbiased function of $\mathbf{T}^{\prime}$. This proves that the statistic $\mathbf{T}^{\prime}$, or equivalently $\mathbf{T}$, is complete for estimating $\mathbf{\Phi}_{\mathbf{A}}$ when the links are asymmetric and both $\alpha$ and $\beta$ are unknown.

Finally, the complete sufficient statistic $\mathbf{T}$ is also minimal owing to Bahadur's theorem which states that if $\mathbf{T}$, taking values in $\Re^{k}$, is sufficient for $\mathbf{\Phi}_{\mathbf{A}}$ and boundedly complete, then $\mathbf{T}$ is minimal sufficient.

What remains is finding an unbiased estimator for $\boldsymbol{\Phi}_{\mathbf{A}}$ as a function of $\mathbf{T}$, which is the MVUE according to the RaoBlackwell-Lehmann-Scheffé theorem. A careful inspection of the sufficient statistics reveals the four unbiased functions of $\mathbf{T}$ for each of $\delta, \phi, \alpha$ and $\beta$ as follows.

$$
\hat{\mathbf{\Phi}}_{\mathbf{A}}=\frac{1}{2(N-1)}\left[\begin{array}{c}
N\left(U_{(1)}+V_{(1)}\right)-(\bar{U}+\bar{V}) \\
N\left(U_{(1)}-V_{(1)}\right)-(\bar{U}-\bar{V}) \\
2 N\left(\bar{U}-U_{(1)}\right) \\
2 N\left(\bar{V}-V_{(1)}\right)
\end{array}\right] .
$$

As a result, the MVUE for the desired parameter, the clock offset, for asymmetric unknown network delays is expressed as

$$
\hat{\phi}_{A}=\frac{1}{N-1}\left[N \frac{U_{(1)}-V_{(1)}}{2}-\frac{\bar{U}-\bar{V}}{2}\right] .
$$

Note that the clock offset estimate depends not only on the minimum order statistics, $U_{(1)}$ and $V_{(1)}$, but also on the mean link delays, $\bar{U}$ and $\bar{V}$.

\section{B. Symmetric Link Delays}

In the symmetric case when $\alpha=\beta \triangleq \lambda$, the likelihood function for the clock offset as a function of observations $\left\{U_{k}\right\}_{k=1}^{N}$ and $\left\{V_{k}\right\}_{k=1}^{N}$ is

$$
\begin{array}{r}
L(\delta, \phi, \lambda)=\lambda^{-2 N} \exp \left[-\frac{1}{\lambda} \sum_{k=1}^{N}\left\{U_{k}+V_{k}-2 \delta\right\}\right] . \\
u\left[U_{(1)}-\delta-\phi\right] \cdot u\left[V_{(1)}-\delta+\phi\right] .
\end{array}
$$


Apparently, for unknown $\lambda$, it seems that $\left\{\sum_{k=1}^{N} U_{k}, U_{(1)}\right.$, $\left.\sum_{k=1}^{N} V_{k}, V_{(1)}\right\}$ are again the sufficient statistics for the estimation of $\boldsymbol{\Phi}_{\mathbf{S}}=\left[\begin{array}{lll}\delta & \phi \lambda\end{array}\right]^{T}$. But then the same sufficient statistics, which have been proved to be complete, can not yield two unbiased estimators. To find the answer of this question, note that (5) can be factored as

$$
\begin{array}{r}
L(\delta, \phi, \alpha, \beta)=g_{1}\left(\sum_{k=1}^{N} U_{k}, \sum_{k=1}^{N} V_{k}, \delta, \lambda\right) g_{2}\left(U_{(1)}, \delta, \phi\right) . \\
g_{3}\left(V_{(1)}, \delta, \phi\right) h_{1}\left(U_{k}, V_{k}\right),
\end{array}
$$

where

$$
\begin{array}{r}
g_{1}\left(\sum_{k=1}^{N} U_{k}, \sum_{k=1}^{N} V_{k}, \delta, \lambda\right)=\lambda^{-2 N} e^{-\frac{1}{\lambda} \sum_{k=1}^{N}\left\{U_{k}+V_{k}-2 \delta\right\}}, \\
g_{2}\left(U_{(1)}, \delta, \phi\right)=u\left[U_{(1)}-\delta-\phi\right], \\
g_{3}\left(V_{(1)}, \delta, \phi\right)=u\left[V_{(1)}-\delta+\phi\right], h_{1}\left(U_{k}, V_{k}\right)=1 .
\end{array}
$$

It turns out that $\mathbf{T}=\left\{\sum_{k=1}^{N}\left(U_{k}+V_{k}\right), U_{(1)}, V_{(1)}\right\}$, and not $\left\{\sum_{k=1}^{N} U_{k}, U_{(1)}, \sum_{k=1}^{N} V_{k}, V_{(1)}\right\}$, is actually the minimal sufficient statistic according to Neymann-Fisher Factorization theorem. Consequently, the clock offset estimator in (4) is not even a choice to consider for not being a function of $\mathbf{T}$.

Now proceeding similarly as before, $\sum_{k=1}^{N}\left(U_{k}+V_{k}\right)$ is dependent on both $U_{(1)}$ and $V_{(1)}$. As a result, $\mathbf{T}$ can be transformed into $\mathbf{T}^{\prime}=\left\{\sum_{k=1}^{N}\left(U_{k}-U_{(1)}+V_{k}-V_{(1)}\right), U_{(1)}, V_{(1)}\right\}$. It is evident from the reasoning in the last subsection that $\sum_{k=1}^{N}\left(U_{k}-U_{(1)}+V_{k}-V_{(1)}\right)$ is Gamma distributed with parameters $(2(N-1), \lambda)$. Hence, $\mathbf{T}^{\prime}$ is a combination of three independent random variables, which in terms of the three parameter Gamma distribution assume the distributions

$$
\begin{gathered}
r=\sum_{k=1}^{N}\left(U_{k}-U_{(1)}+V_{k}-V_{(1)}\right) \sim \Gamma(2(N-1), \lambda, 0), \\
U_{(1)} \sim \Gamma(1, \lambda / N, \delta+\phi), \quad V_{(1)} \sim \Gamma(1, \lambda / N, \delta-\phi) .
\end{gathered}
$$

Next, defining $g\left(\mathbf{T}^{\prime}\right)$ and $h\left(\mathbf{T}^{\prime}\right)$ as functions of $\mathbf{T}^{\prime}$ such that $E\left[g\left(\mathbf{T}^{\prime}\right)\right]=E\left[h\left(\mathbf{T}^{\prime}\right)\right]=\mathbf{\Phi}_{\mathbf{S}}$,

$$
E\left[g\left(\mathbf{T}^{\prime}\right)-h\left(\mathbf{T}^{\prime}\right)\right]=E\left[\pi\left(\mathbf{T}^{\prime}\right)\right]=0 \quad \forall \mathbf{\Phi}_{\mathbf{S}}
$$

where the expectation is taken with respect to $p_{\mathbf{T}^{\prime}}\left(\mathbf{T}^{\prime} ; \mathbf{\Phi}_{\mathbf{S}}\right)$. As a result, since the domain of $r$ and $s$ are controlled by $U_{(1)}$ and $V_{(1)}$, respectively

$$
\begin{array}{r}
\iint_{R_{U_{(1)}}, V_{(1)}} \pi\left(r, U_{(1)}, V_{(1)}\right) \cdot \frac{\lambda^{-\{2(N-1)\}}}{\Gamma[2(N-1)]} r^{2 N-3} e^{-\frac{r}{\lambda}} \cdot\left(\frac{N}{\lambda}\right)^{2} \\
e^{-\frac{N}{\lambda}\left\{U_{(1)}+V_{(1)}-2 \delta\right\}} \cdot d r d U_{(1)} d V_{(1)}=0 \quad \forall \mathbf{\Phi}_{\mathbf{S}}
\end{array}
$$

where $R_{U_{(1)}, V_{(1)}}$ is the region defined by $u\left[U_{(1)}-\delta-\phi\right]$ and $u\left[V_{(1)}-\delta-\phi\right]$. It follows that

$\int_{-\infty}^{\infty} \int_{-\infty}^{\infty} \int_{-\infty}^{\infty} \pi\left(r, U_{(1)}, V_{(1)}\right) \cdot r^{2 N-3} e^{-\frac{N}{\lambda}\left\{\frac{r}{N}+U_{(1)}+V_{(1)}\right\}}$
$d r d U_{(1)} d V_{(1)}=0 \quad \forall \mathbf{\Phi}_{\mathbf{S}}$

From the uniqueness theorem for the two-sided Laplace transform, it follows that $\pi\left(\mathbf{T}^{\prime}\right)=0$ almost everywhere, resulting in the completeness of $\mathbf{T}^{\prime}$, or equivalently $\mathbf{T}$. Hence, $\mathbf{T}$ is also the minimal sufficient statistics from Bahadur's theorem and the MVUE is expressed as

$$
\hat{\mathbf{\Phi}}_{\mathbf{S}}=\frac{1}{2(N-1)}\left[\begin{array}{c}
N\left(U_{(1)}+V_{(1)}\right)-(\bar{U}+\bar{V}) \\
(N-1)\left(U_{(1)}-V_{(1)}\right) \\
N\left\{(\bar{U}+\bar{V})-\left(U_{(1)}+V_{(1)}\right)\right\}
\end{array}\right] .
$$

Hence, the MVUE for the clock offset, in the case of symmetric unknown network delays, is expressed as

$$
\hat{\phi}_{S}=\frac{U_{(1)}-V_{(1)}}{2} .
$$

It is evident that the MVUE in symmetric delay case coincides with the MLE in (1), which further advocates the use of the MLE to estimate the clock offset in symmetric conditions.

\section{CONClusions AND Future Work}

In this paper, the minimum variance unbiased estimate for the clock offset between two nodes running a sender-receiver time synchronization protocol is derived. The result is an important contribution since it also takes into account the mean link delays in addition to the minimum link delays for reducing the variance in asymmetric delay case. It also shows that the maximum likelihood estimator and the minimum variance unbiased estimator have the same expression in symmetric link delays. Additionally, the clock synchronization technique derived here is also more generally applicable in addition to the protocols in the sensor networks.

As a future work, solving the global clock synchronization problem is an interesting research area. The estimation of the clock offset over multiple number of hops and the subsequent error accumulation is important for engineers for the design and analysis of an optimal global clock synchronization algorithm.

\section{REFERENCES}

[1] D. Mills, "Internet time synchronization: The network time protocol," IEEE Transactions on Communications, Vol. 39, No. 10, pp. 1482-1493, Oct 1991.

[2] K. Romer, "Time Synchronization in Ad Hoc Networks," Proceedings of ACM Symposium on Mobile Ad Hoc Networking and Computing, pp. 173182, Oct 2001.

[3] J. Elson, L. Girod and D. Estrin, "Fine-Grained Network Time Synchronization using Reference Broadcasts," In Proceedings of the 5th Symposium on Operating System Design and Implementation, Boston, Massachusetts, Dec 2002.

[4] S. Ganeriwal, R. Kumar and M.B. Srivastava, "Timing Synch Protocol for Sensor Networks," In Proceedings of 1st International Conference on Embedded Network Sensor Systems, Los Angeles, CA, Nov 2003.

[5] W. Su, and I. F. Akyildiz, "Time-diffusion synchronization protocol for wireless sensor networks," IEEE/ACM Transactions on Networking, Volume 13, No. 2, pp. 384- 397, Apr 2005.

[6] H. S. Abdel-Ghaffar, "Analysis of synchronization algorithm with timeout control over networks with exponentially symmetric delays," IEEE Transactions on Communications, Vol. 50, pp. 1652-1661, Oct 2002.

[7] D. R. Jeske, "On Maximum Likelihood Estimation of Clock Offset," IEEE Transactions on Communications, Vol. 53, No. 1, pp. 53-54, Jan 2005.

[8] S. M. Kay, Fundamentals of Statistical Signal Processing, Vol. I. Estimation Theory, Prentice Hall, 1993. 